\title{
Estrous Correlated Modulations of Circadian and Ultradian Wheel-Running Activity Rhythms in LEW/Ztm Rats
}

\author{
FRANZISKA WOLLNIK ${ }^{1}$ AND FRED W. TUREK \\ Department of Neurobiology and Physiology, Northwestern University, Evanston, IL 60208
}

Received 16 November 1987

\begin{abstract}
WOLLNIK, F. AND F. W. TUREK. Estrous correlated modulations of circadian and ultradian wheel-running activity rhythms in LEW/Ztm rats. PHYSIOL BEHAV 43(3)389-396, 1988.-Estrogen treatment alters the expression of ultradian activity rhythms in male and female LEW/Ztm rats. This finding raises the possibility that the expression of ultradian rhythms may vary on different days of the estrous cycle. To test this hypothesis, we recorded the circadian and ultradian wheel-running activity rhythms of entrained (LD 12:12) and free-running sexually mature LEW/Ztm females during their 4- or 5-day estrous cycle. The mean daily activity, the duration of activity, the circadian period of activity, and the occurrence of ultradian rhythms differed significantly among the days of the estrous cycle. In LD 12:12, the phase angle difference between the beginning of activity and light offset varied reliably in 5-day cycling animals. The highest daily mean of activity, the longest duration, and the shortest circadian period length were observed on the day of estrus in both entrained and free-running animals. The day of estrus was characterized by a constant high level of activity throughout the activity phase, while the days following ovulation showed a bi- or trimodal activity pattern. Power spectrum analysis revealed significant ultradian components for the days of metestrus and diestrus, but only circadian components for the days of proestrus and estrus. These results were interpreted as indicating that endogenous changes in circulating hormone levels can induce changes in the ultradian and circadian patterns of wheel-running activity in LEW/Ztm rats.
\end{abstract}

Wheel-running activity Estrous cycle Ultradian and circadian rhythms Laboratory rat

STEROID hormones have been reported to modulate the amplitude, phase, and period of mammalian circadian rhythms (for review see $[25,30]$ ). In female hamsters and rats, the time of activity onset fluctuates with the estrous cycle in animals entrained to a light-dark cycle as well as in animals maintained under constant illumination or darkness $[2,7,14,22]$. Activity onset in hamsters occurs earlier on both proestrus and estrus, the days of the estrous cycle when serum levels of estrogen are high [5], whereas in rats the activity onset is earlier only on the day of estrus [2]. Exogenously supplied gonadal hormones also alter the periodicity of circadian rhythms. Silastic estrogen-filled capsules that produce proestrous levels of estrogen shorten the period of the free-running rhythms in ovariectomized rats and hamsters $[1,14,31]$, and increase the total amount of wheelrunning activity $[11,22]$. Although progesterone alone does not affect circadian rhythms $[14,17,22]$, it can antagonize estrogen's action on activity rhythms in rats and hamsters [3, $4,17,22$ ]. It is likely that the interaction of progesterone and estrogen modulates the circadian rhythm of activity in female rats and hamsters on a day-to-day basis $[2,22]$.

Recent studies indicate that gonadal hormones can also alter the expression of ultradian rhythms in male and female rats of the inbred strain $\mathrm{LEW} / \mathrm{Ztm}$. Male $\mathrm{LEW} / \mathrm{Ztm}$ rats show a very precise and reproducible trimodal locomotor activity pattern that exhibits substantial heritability with a general recessive mode of inheritance [28]. The trimodal activity pattern generates ultradian peaks in the range of 4 and 4.8 hours when tested with different methods of period analysis [6,27]. Gonadally intact $\mathrm{LEW} / \mathrm{Ztm}$ females do not show ultradian rhythmicity [26], but following ovariectomy, they exhibit ultradian rhythms similar to those shown by males [27]. Estrogen released continuously from long-term subcutaneous implants generally prevents the expression of ultradian activity rhythms in ovariectomized females as well as in castrated and noncastrated males [27]. The finding that estrogen prevents the expression of ultradian rhythms on a long-term basis has led to the question whether endogenous changes of circulating hormone levels associated with the estrous cycle can induce changes in the expression of ultradian rhythms on a day-to-day basis.

The purpose of the present study was to examine the ultradian and circadian patterns of wheel-running activity of sexually mature LEW/Ztm female rats. Data of both entrained and free-running animals were analyzed for each day of the estrous cycle in order to determine whether estrous condition influences not only circadian but also ultradian parameters of the activity rhythm.

\footnotetext{
${ }^{1}$ Requests for reprints should be addressed to Dr. Franziska Wollnik, Universität Konstanz, Fakultät für Biologie, Postfach 55 60, D-7750 Konstanz 1, F.R.G.
} 
METHOD

\section{Animals}

LEW/Ztm female rats, originally obtained from Central Animal Laboratory, Medical School, Hannover, West Germany, were bred and raised in our own laboratory on a light-dark cycle of $12 \mathrm{hr}$ light (LD 12:12, lights on at 0600). At 80 days of age, 20 female rats were placed in individual cages containing an activity wheel and a small adjacent housing compartment. The animals were housed in a room maintained under LD 12:12, and two days before the start of the experiment half the animals were blinded (bilateral orbital enucleation) under anesthesia (100 mg/kg Ketamine Hydrochloride $+10 \mathrm{mg}$ Xylazin, IM) to allow for examination of free-running activity rhythms. The room also contained mature LEW/Ztm male rats. All animals had free access to food and water. The room was entered once every day for animal maintenance and for recording of vaginal smears. In 3 sighted and 3 blinded animals vaginal smears were taken daily during the light period. The rest of the animals remained undisturbed, except for cage cleaning once a week.

\section{Data Collection and Analysis}

Wheel-running activity was monitored on line with a microcomputer (Apple $\mathrm{II}+$ ) and was recorded as number of wheel revolutions per $5 \mathrm{~min}$. Data were stored on floppy disk every $24 \mathrm{hr}$. All subsequent calculations were based on these 5 -min values. Event records were printed at 15 -min intervals over 24 hr. (Figs. 1A, 4A). Total counts of wheel revolutions for each 15-min interval are shown as a histogram. Maximum height of the histogram represents more than 150 counts (i.e., an average of 10 counts/min), while a blank space indicates fewer than 15 counts (i.e., an average of 1 count $/ \mathrm{min}$ ).

The estrous cycle was monitored in 3 sighted and 3 blinded female rats by vaginal smears. The day of estrus, which is characterized by a conspicuous vaginal discharge, was defined as the $24-\mathrm{hr}$ interval starting at 1200 (noon) on the day before ovulation and ending at 1200 on the next morning. Using this method, the complete activity phase of an animal was included in each 24 -hr data set. For the 14 animals in which daily vaginal smears were not taken, the day of estrus was defined as the day that contained the peak number of wheel revolutions over a 4- to 5-day cycle. In entrained animals the days of the estrous cycle were referenced to the entraining LD cycle. The day of metestrus, for example, was the $24-\mathrm{hr}$ interval between 1200 on the day following the peak number of wheel revolutions and 1200 of the next day. In the free-running animals the days of the estrous cycle were defined with respect to their mean freerunning period. The day of estrus included the activity-time containing the peak number of revolutions and one half of both the preceding and following rest interval.

Statistical analyses were applied to data sets of 6 or 7 estrous cycles for each animal. The presence of circadian and ultradian rhythms was tested by the "generalized harmonic spectral analysis" $[12,16]$ and the "chi square periodogram" [20]. Both methods have proven to be reliable for the detection of ultradian periodicities in the presence of circadian rhythms [27]. Nevertheless, the results of the two methods were compared to check the reliability of each method. Statements about the periodicity of each animal were based on the application of both methods.

Measures of circadian periodicity included: (1) mean daily activity: the average number of wheel revolutions for all 5-min intervals within a $24-\mathrm{hr}$ period; (2) duration: the total time span of all 5-min intervals within a 24 -hr interval containing more than 5 revolutions; (3) phase angle difference: the phase relationship between onset of activity and onset of the dark phase of the LD cycle; and (4) period $(\tau)$ : the length of one complete activity cycle. Period was calculated from the activity onset of the preceding day to the activity onset of the reference day, the onset of activity being defined as the time of the first of six consecutive 5-min intervals of activity in which each interval contained at least 5 revolutions. The chi square periodogram also yields estimates of $\tau$ for different days of the estrous cycle, which are quite similar to those obtained by measuring the length between two activity onsets. For the statistical analysis, however, the estimates from the periodogram were not used because they provide only one estimate for 6 or 7 estrous cycles and give no information about the variation between the individual cycles. Statistical significance was ascertained by two-way analysis of variance (subject $\times$ trial, 6 or 7 estrous cycles for each individual animal) and Scheffe's multiple $t$-test.

\section{RESULTS}

Of the $20 \mathrm{LEW} / \mathrm{Ztm}$ females used in this study 10 entrained and 7 free-running rats showed a clear estrous cycle of either 4 or 5 days. These 17 animals were selected for further analysis.

\section{LD 12:12 Entrained Animals}

Female LEW/Ztm rats entrained to LD 12:12 showed a clear modification of the activity pattern and differences in the level of activity during the estrous cycle. Data from a representative $\mathrm{LEW} / \mathrm{Ztm}$ female with a 5-day cycle are shown in Fig. 1. Typically, intense continuous activity occurred during the active phase on the days of proestrus and estrus. The level of activity decreased and the activity pattern fragmented on the days after ovulation. The average amount of activity was lower on metestrus and diestrus than on estrus, and a bi- or trimodal activity pattern was apparent on metestrus and diestrus but not on estrus. Periodogram and power spectrum analyses showed statistically significant ultradian components with periods of $4,4.8$, and $6 \mathrm{hr}$ for the days of metestrus and diestrus 1 and 2 , whereas on proestrus and estrus only circadian components were significant.

The individual power spectra were pooled for the 5-day or 4-day cycling rats and are shown in Fig. 2A,B. Statistical comparison of the amplitudes of spectral estimates revealed significant differences between days for the 4-, 4.8- and 6-hr spectral estimates. The highest amplitudes in the ultradian range were found for the 4.8 -hr spectral estimates on the days of metestrus and diestrus. The corresponding spectral estimates on proestrus and estrus were significantly lower. No significant variations were found for the amplitudes of the 24-hr spectral estimates.

Measures of circadian rhythmicity, such as mean daily activity, duration, and period, varied significantly across days of the 4- and 5-day estrous cycle (Fig. 3). The phase angle between lights off and activity onset differed significantly between days for the 5-day cycling rats. The phase angle for the 4-day cycling rats showed a similar variation, but no statistically significant difference across days. The lack of significance in this group is most likely due to the small number of animals. In either 4- or 5-day cycling 

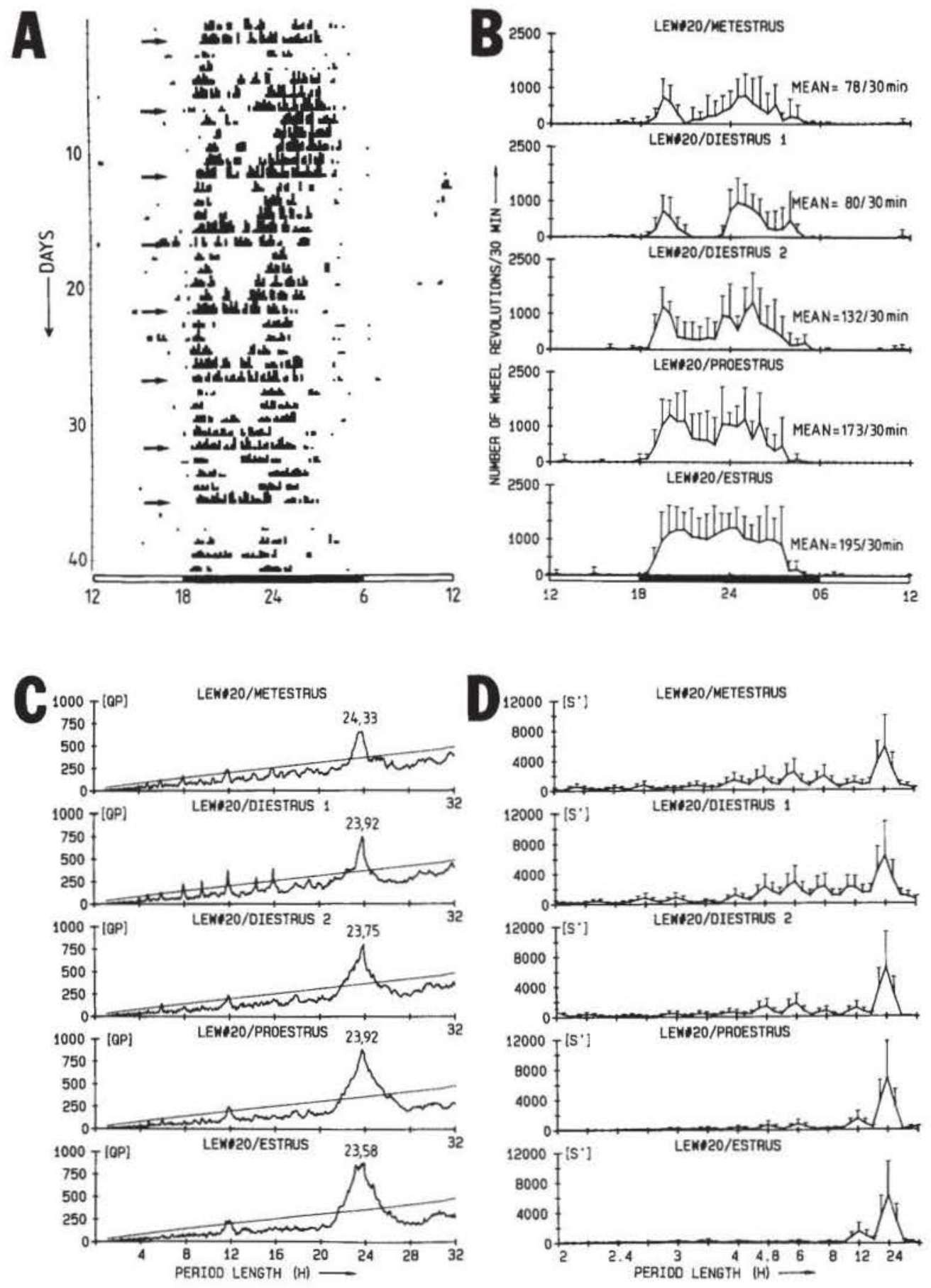

FIG. 1. Estrous correlated modulation in the activity pattern of a 5-day cycling LEW/Ztm female rat entrained to LD 12:12. (A) Wheel-running activity record. Numbers on the ordinate indicate days, and hours within the day are shown on the abscissa. Estrous cycle was monitored by vaginal smears and estrous days are indicated by arrows. (B) Average distribution of activity during the estrous cycle. Thirty-min mean values are plotted over time with standard deviations between days $(n=7)$ shown as vertical lines. (C) Chi square periodogram analysis revealed significant ultradian rhythms with a period of $4 \mathrm{hr}$ for metestrus, 4 and $4.8 \mathrm{hr}$ for diestrus 1 , and $6 \mathrm{hr}$ for diestrus 2 . The sloping line represents a significance level of $p<0.01$. A series of multiple ultradian peaks is interpreted as being caused by only the largest common submultiple of all those peaks. For more detailed information on the interpretation of chi square periodograms see $[20,27]$. (D) Spectral analysis of the wheel-running activity. The $95 \%$ confidence limits of spectral estimates are plotted as vertical lines. 

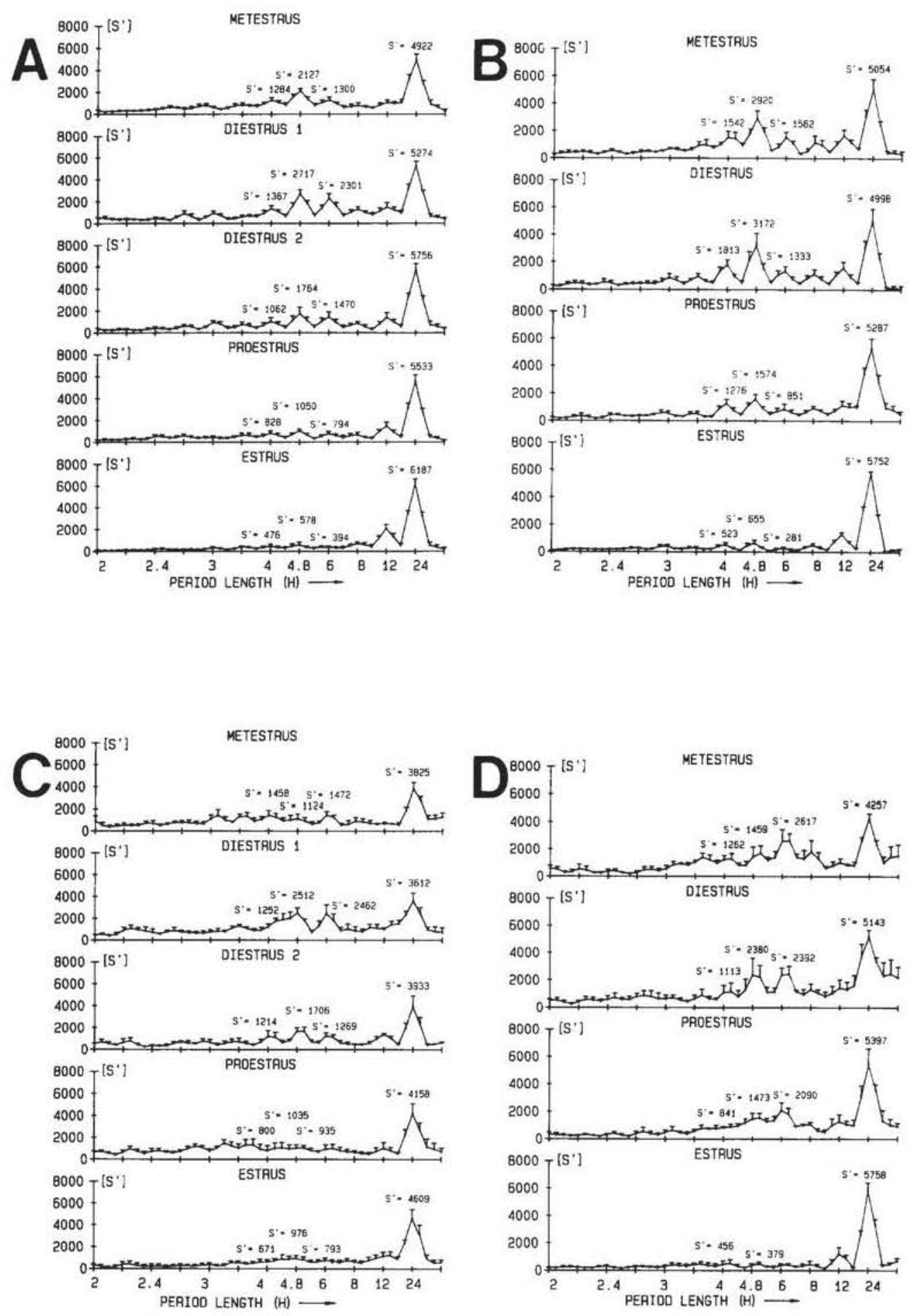

FIG. 2. Pooled spectral analysis of wheel-running activity rhythms in entrained $(A, B)$ and free-running (C,D) LEW/Ztm female rats with either 5-day $(A, C)$ or 4-day (B,D) cycles. The $95 \%$ confidence limits of spectral estimates are plotted as vertical lines. A one-way ANOVA was used to verify significant differences in the amplitude of spectral estimates. Differences between days were significant for the 4-, 4.8-, and 6-hr spectral estimates. LD 12:12, 5-day: $\mathrm{F}_{6-\mathrm{hr}}=13.27, p<0.01 ; \mathrm{F}_{4.8-\mathrm{hr}}=10.97, p<0.01 ; \mathrm{F}_{4-\mathrm{hr}}=3.91$, $p<0.05 ;$ LD 12:12, 4-day: $\mathrm{F}_{6-\mathrm{hr}}=17.25, p<0.01 ; \mathrm{F}_{4.8-\mathrm{hr}}=22.71, p<0.01 ; \mathrm{F}_{4-\mathrm{hr}}=9.69, p<0.01$ : free-running, 5-day: $\mathrm{F}_{6-\mathrm{hr}}=6.36, p<0.01 ; \mathrm{F}_{4.8-\mathrm{hr}}=21.35, p<0.01 ; \mathrm{F}_{4-\mathrm{hr}}=4.35, p<0.05$; free-running, 4-day: $\mathrm{F}_{6-\mathrm{hr}}=20.88$, $p<0.01 ; \mathrm{F}_{4.8 \cdot h r}=13.02, p<0.01 ; \mathrm{F}_{4 \cdot h r}=4.71, p<0.05$. 

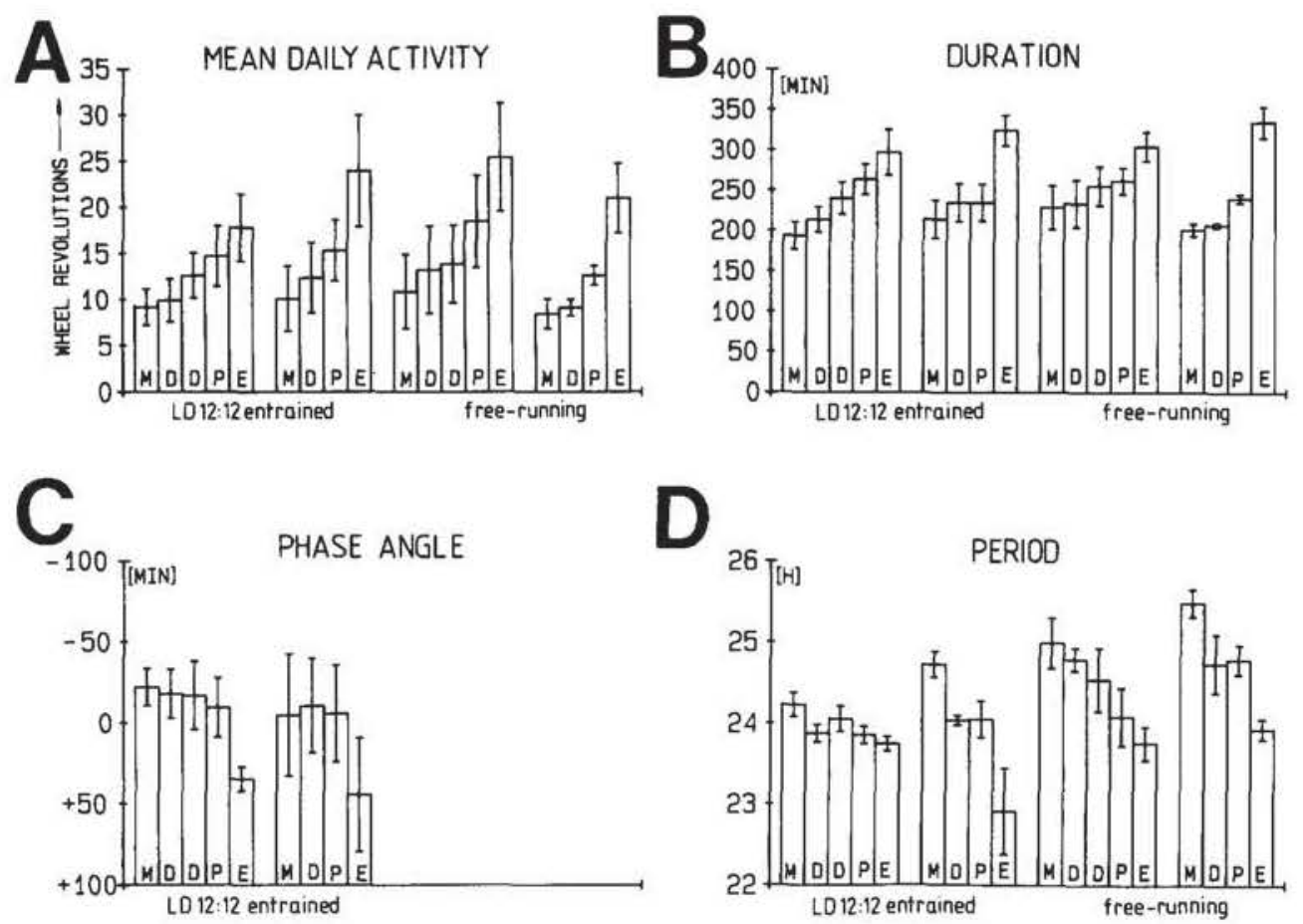

FIG. 3. Parameters of circadian rhythmicity during the 4- or 5-day estrous cycle of LD 12:12 entrained and free-running LEW/Ztm rats. The histograms show means \pm SEM of $\mathrm{n}$ animals for each day of the estrous cycle $(M=$ Metestrus, $D=$ Diestrus, $P=$ Proestrus, $E=$ Estrus; $L D$ 12:12 5-day: $n=6$, 4 -day: $n=4$; freerunning 5-day: $n=4$, 4-day: $n=3$ ). (A) Mean daily activity showed statistically significant differences for LD 12:12 5-day: $\mathrm{F}(4,20)=21.48, p<0.01 ; 4$-day: $\mathrm{F}(3,9)=33.81, p<0.01$; free-running 5-day: $\mathrm{F}(4,12)=11.08$, $p<0.01$; 4-day: $F(3,6)=16.26, p<0.01$. (B) Duration of activity showed statistically significant differences for LD 12:12 5-day: $\mathbf{F}(4,20)=20.17, p<0.01$; 4-day: $\mathbf{F}(3,9)=14.13, p<0.01$; free-running 5-day: $\mathrm{F}(4,12)=8.93, p<0.01 ; 4$-day: $\mathrm{F}(3,6)=16.87, p<0.01$. (C) Phase showed statistically significant differences for LD 12:12 5-day: $F(4,20)=20.39, p<0.01$. A negative value indicates activity begun after the onset of dark. (D) Period showed statistically significant differences for LD 12:12 5-day: $\mathrm{F}(4,20)=3.69, p<0.05$; 4-day: $\mathrm{F}(3,9)=8.29, p<0.01$; free-running 5-day: $\mathrm{F}(4,12)=3.91, p<0.05 ; 4$-day: $\mathrm{F}(3,6)=4.87, p<0.05$.

animals, the most distinctive differences were found on the day of estrus. The average number of wheel revolutions on the day of estrus (5-day: $18 \pm 4$ rev. $/ 5 \mathrm{~min}$; 4 -day: $24 \pm 6 \mathrm{rev} . / 5$ $\mathrm{min}$ ) was approximately two times higher than on the day of metestrus (5-day: $9 \pm 2 \mathrm{rev} . / 5 \mathrm{~min}$; 4 -day: $11 \pm 4 \mathrm{rev} . / 5 \mathrm{~min}$ ). Duration of activity was over $100 \mathrm{~min}$ longer on estrus (5day: $297 \pm 28 \mathrm{~min}$; 4-day: $324 \pm 19 \mathrm{~min}$ ) than on metestrus (5-day: $194 \pm 17 \mathrm{~min}$; 4-day: $214 \pm 24 \mathrm{~min}$ ).

Only on the day of estrus was activity initiated before the onset of the dark phase resulting in positive values for the phase angle. In 5-day cycling rats, the mean time of activity onset on the day of estrus was 44 to 57 min earlier than on the other days of the estrous cycle. A similar relationship was found in the 4-day cycling rats (49 to $55 \mathrm{~min}$ ). The period of the entrained circadian activity rhythm was shortest on estrus (5-day: $23.73 \pm 0.09 \mathrm{hr}$; 4-day: $22.91 \pm 0.53 \mathrm{hr}$ ) and longest on metestrus (5-day: $24.23 \pm 0.15 \mathrm{hr}$; 4 -day: $24.73 \pm 0.16 \mathrm{hr}$ ). Although large differences in the period were observed among each of the 4- or 5-day cycling rats, the period averaged over all days of the cycle was almost exactly the same as the period of the entraining light-dark cycle, i.e., $23.95 \pm 0.05 \mathrm{hr}$ for 5 -day and $23.93 \pm 14 \mathrm{hr}$ for 4-day cycling animals.

\section{Free-Running Animals}

Regular cycles of about 4 or 5 days persisted in 7 of 10 animals after they were blinded. The 3 females omitted from the analysis showed a circadian activity pattern, without detectable variations in intervals of about 4 or 5 days. The data from a representative LEW/Ztm female with a 4-day cycle are shown in Fig. 4. The pattern of wheel-running activity during the days of the estrous cycle was similar to that seen under LD 12:12 (Fig. 1). The average number of wheel revolutions was highest on the day of estrus and the activity pattern changed from a unimodal pattern on the day of estrus to a trimodal pattern on the day of diestrus. Power spectrum and periodogram analyses detected significant ultradian components with periods in the range of 4 to $6 \mathrm{hr}$ for the day of diestrus. The shortest circadian period was observed on the day of estrus $(\tau=23.83 \mathrm{hr})$, and the longest on the day of metestrus $(\tau=24.92 \mathrm{hr})$.

The pooled power spectra (Fig. 2C,D) show ultradian components with periods ranging from 4 to $6 \mathrm{hr}$ for the days of metestrus and diestrus. The highest amplitudes in the ultradian range were found for the 4.8- and 6-hr spectral estimates on the days of metestrus and diestrus. The amplitudes 

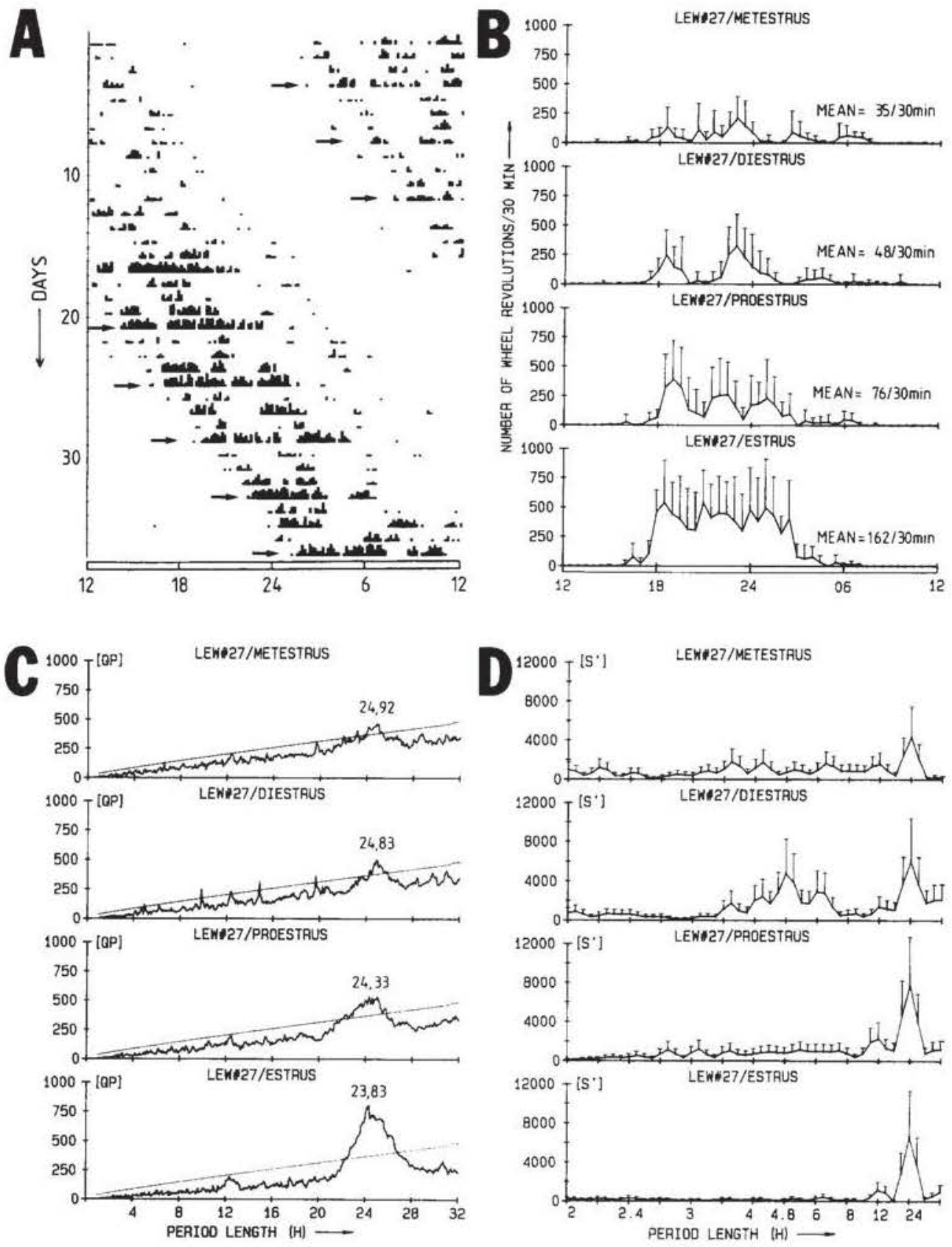

FIG. 4. Estrous cycle correlated modulation in the activity pattern of a 4-day cycling LEW/Ztm female rat maintained under free-running conditions. (A) Wheel-running activity record. A 4-day modulation of the activity pattern occurred in synchrony with the estrous cycle. (B) Average distribution of activity during the estrous cycle. Thirty-min mean values are plotted over time with standard deviations between days shown as vertical lines. (C) Chi square periodogram analysis. (D) Spectral analysis.

of corresponding spectral estimates on the day of estrus were significantly lower. No significant variations were found for the amplitudes of the 24-hr spectral estimates.

Statistically significant differences in mean daily activity and duration existed among the days of the estrous cycle (Fig. 3). The highest level of activity (5-day: $25 \pm 6 \mathrm{rev} . / 5 \mathrm{~min}$; 4-day: $21 \pm 4 \mathrm{rev} . / 5 \mathrm{~min}$ ) and the longest duration of activity (5-day: $305 \pm 18 \mathrm{~min}$; 4-day: $335 \pm 19 \mathrm{~min}$ ) were found on the day of estrus, the lowest activity level (5-day: $11 \pm 4 \mathrm{rev} . / 5$ min; 4-day: $8 \pm 2 \mathrm{rev} . / 5 \mathrm{~min}$ ) and the shortest duration of activity on the day of metestrus. The period was shortest on the day of estrus (5-day: $23.75 \pm 0.21 \mathrm{hr}$; 4 -day: $23.93 \pm 0.13$ $\mathrm{hr}$ ) and longest on metestrus (5-day: $24.99 \pm 0.31 \mathrm{hr}$; 4-day: $25.48 \pm 0.17 \mathrm{hr}$ ). The period averaged over all days of the cycle was $24.42 \pm 0.57 \mathrm{hr}$ for the 5 -day and $24.72 \pm 0.64 \mathrm{hr}$ for the 4-day cycling rats. 


\section{DISCUSSION}

The results of the present study demonstrate that the wheel-running activity pattern in sexually mature LEW/Ztm rats varies systematically with the estrous cycle in both entrained and free-running LEW/Ztm rats. The important finding of this study is an estrous correlated modulation in the expression of ultradian rhythms in wheel-running activity. The day of estrus was characterized by a constant high level of activity throughout the active phase, whereas on the days of metestrus and diestrus the total amount of activity decreased and bi- or trimodal activity patterns were apparent. Power spectrum and periodogram analyses detected significant ultradian components in the range from 4 to $6 \mathrm{hr}$ for the days of metestrus and diestrus, whereas on the days of proestrus and estrus only circadian components were found. The present study also showed systematic changes of circadian parameters during the estrous cycle which are consistent with previous findings in rats [2]. Both entrained and free-running animals with 4- and 5-day cycles had the shortest period, the longest duration, and the highest mean level of activity on the day of estrus. Furthermore, activity onset occurred earlier on the day of estrus in animals entrained to LD 12:12.

The present results suggest that estrous correlated variations in the ultradian patterns result from alterations in hormone levels during the estrous cycle. It has recently been shown that estrogen released from long-term subcutaneous implants generally prevents the expression of ultradian activity rhythms in ovariectomized females as well as in castrated and noncastrated males of the LEW/Ztm strain [27]. In this previous study, however, no evidence was found for an estrous correlated modulation of the activity pattern in intact $\mathrm{LEW} / \mathrm{Ztm}$ rats. This was most likely due to the Automex-like system previously used for the measurement of overall locomotor activity. Running wheel recordings seem to be more suitable for measuring changes in parameters of circadian and ultradian rhythms. Other investigations on estrous correlated modulations of wheel-running activity in other laboratory rats $[2,10,11]$ did not specifically mention the aspect of ultradian rhythms in their data. However, visual inspection of their wheel-running activity records from female rats also show bi- or trimodal activity patterns correlated with the days of metestrus and diestrus, while the days of proestrus and estrus ivere characterized by an almost constant plateau of activity. The present studies on LEW/Ztm rats describe and quantify these changes in ultradian parameters for the first time.

The pattern of estrogen level changes during the estrous cycle of rats [15] is consistent with, and sufficient to explain, the patterns of ultradian rhythmicity observed during the estrous cycle. Serum levels of estrogen are equally high during the night of proestrus and estrus [15], and no ultradian rhythms were observed on those two days. However, it is known that exogenously supplied estrogen has long aftereffects on the circadian system of hamsters [14,22]. These after-effects appear to be too long for the day-to-day changes seen during a normal estrous cycle. Because progesterone is known to reverse the action of estrogen on the circadian activity pattern in less than 24 hours [3, 4, 22], several authors $[2,22]$ have suggested that estrogen and progesterone interact to yield the modulation of circadian rhythmicity during the estrous cycle of rats and hamsters. So far, no data are available on after-effects of estrogen or a possible antagonizing effect of progesterone on the ultradian activity pattern of $\mathrm{LEW} / \mathrm{Ztm}$ rats. Therefore, we cannot exclude the possibility that progesterone is also involved in the modulation of the ultradian activity pattern in this inbred strain.

The mechanism by which estrogen or other gonadal hormones change the expression of ultradian rhythms in the activity pattern of $\mathrm{LEW} / \mathrm{Ztm}$ rats is not known. One possible explanation for the fact that ultradian rhythms are not detectable on the days of proestrus and estrus is that they might be masked by the high levels of activity. This explanation assumes that ultradian and circadian components of wheelrunning activity are regulated by independent pacemaker systems. However, a recent study on the effect of suprachiasmatic nuclei (SCN) lesions on the wheel-running activity pattern of LEW/Ztm rats [29] showed that both circadian and ultradian rhythms are regulated by the SCN. Complete $\mathrm{SCN}$ lesions resulted in a complete loss of circadian and ultradian components of the activity rhythm. An alternative explanation for estrous correlated changes in the expression of ultradian rhythms is derived from the multioscillatory nature of the mammalian circadian pacemaker system [18, 19, 23 ]. This explanation assumes that the ultradian rhythms reflect the activity of several circadian oscillators coupled to each other in an estrogen dependent phase relationship. It is known that hormones, especially estrogen, can strengthen the coupling between circadian oscillators. For example, the circadian activity rhythm of hamsters exposed to constant illumination dissociates or splits into two circadian components [24] and administration of estrogen can decrease the incidence of splitting in ovariectomized hamsters [13]. In this latter study, estrogen also inhibited other forms of dissociation of the circadian activity pattern characterized by ultradian peaks in the periodogram. Daan et al. [9] proposed that hormones (in this case testosterone) might influence the period of circadian rhythms by strengthening or weakening the synchrony between different oscillators. The present data show similar changes during the estrous cycle. The activity records (Fig. 1A, 4A) and the average daily profiles (Fig. 1B, 4B) show a different phase relationship between the separate activity bouts during the estrous cycles. The bi- or trimodal activity components are further apart from each other on the days of metestrus and diestrus 1 , and they move closer together on the days of diestrus 2 and proestrus.

In conclusion, the presently available data on $\mathrm{LEW} / \mathrm{Ztm}$ rats $([27,29]$ and present results) suggest that the bi- or trimodal activity pattern observed in males and ovariectomized females of this strain reflects the activity of several weakly coupled circadian oscillators. Estrogen may change the phase relationship between those oscillators not only under long-term administration, but also on a day-to-day basis during the normal estrous cycle.

\section{ACKNOWLEDGEMENTS}

This research was supported by a grant from Deutsche Forschungsgemeinschaft (II 02-Wo $354 / 2-1$ ) to Franziska Wollnik and by NIH grant HD-09885 to Fred W. Turek. 


\section{REFERENCES}

1. Albers, H. E. Gonadal hormones organize and modulate the circadian system of the rat. Am. J. Physiol. 241:R62-R66; 1981.

2. Albers, H. E.; Gerall, A. A.; Axelson, J. F. Effect of reproductive state on circadian periodicity in the rat. Physiol. Behav. $26: 21-25 ; 1981$.

3. Axelson, J. F.; Gerall, A. A.; Albers, H. E. The effects of progesterone on the amplitude, periodicity and onset of estrous activity cycles in the intact female rat. Biol. Reprod. 20:76 A 1979.

4. Axelson, J. F.; Gerall, A. A.; Albers, H. E. Effects of progesterone on the estrous activity cycle of the rat. Physiol. Behav. 26:631-635; 1981.

5. Baranczuk, R.; Greenwald, G. S. Peripheral levels of estrogen in the cyclic hamster. Endrocrinology 92:805-812; 1973.

6. Büttner, D.; Wollnik, F. Strain-differentiated circadian and ultradian rhythms in locomotor activity of the laboratory rat. Behav. Genet. 14:137-152; 1984.

7. Campbell, C. S.; Turek, F. W. Cyclic function of the mammalian ovary. In: Aschoff, J., ed. Handbook of behavioral neurobiology vol. 4: Biological rhythms. New York: Plenum Press; 1981:523-545.

8. Carter, S. B. The relationship of the time of ovulation to the activity pattern in the rat. J. Endocrinol. 53:333-334; 1972.

9. Daan, S.: Damassa, D.; Pittendrigh, C. S.; Smith, E. R. An effect of castration and testosterone replacement on a circadian pacemaker in mice (Mus musculus). Proc. Natl. Acad. Sci USA 72:3744-3747; 1975 .

10. Gentry, R. T.; Wade, G. N. Sex differences in sensitivity of food intake, body weight, and running-wheel activity to ovarian steroids in rats. J. Comp. Psychol. 90:747-754; 1976.

11. Gerall, A. A.; Napoli, A. M.; Cooper, U. C. Daily and hourly estrous running in intact spayed and estrone implanted rats. Physiol. Behav. 10:225-229; 1973.

12. Halberg, F.; Panofsky, H. Thermo-variance spectra; methods and clinical illustrations. Exp. Med. Surg. 19:285-321; 1961

13. Morin, L. P. Effect of ovarian hormones on synchrony of hamster circadian rhythms. Physiol. Behav. 24:741-749; 1980.

14. Morin, L. P.; Fitzgerald, K. M.; Zucker, I. Estradiol shortens the period of hamster circadian rhythms. Science 196:305-307; 1977.

15. Nequin, L. G.; Alvarez, J.; Schwartz, N. B. Steroid control of gonadotropin release. J. Steroid Biochem. 6:1007-1012; 1975.

16. Panofsky, H.; Halberg, F., II. Thermo-variance spectra; simplified computational example and other methology. Exp. Med. Surg. 19:322-338; 1961 .

17. Rodier, W. K. Progesterone-estrogen interactions in the control of activity-wheel running in the female rat. J. Comp. Physiol. Psychol. 74:365-373; 1971.
18. Rosenwasser, A. M.; Adler, N. T. Structure and function in circadian timing systems: evidence for multiple coupled circadian oscillators. Neurosci. Biobehav. Rev. 10:431-448; 1986.

19. Rusak, B. Neural mechanisms for entrainment and generation of mammalian circadian rhythms. Fed. Proc. 38:2589-2595; 1979.

20. Sokolove, P. G.; Bushel, W. N. The chi square periodogram: its utility for analysis of circadian rhythms. J. Theor. Biol. 72:131$160 ; 1978$.

21. Sridaran, R.; McCormack, C. E. Predicting the time of ovulation in rats by monitoring running activity. Fed. Proc. 36:313, Abstr. 226; 1977.

22. Takahashi, J. S.; Menaker, M. Interaction of estradiol and progesterone: effects on circadian locomotor rhythm of female golden hamsters. Am. J. Physiol. 239:R497-R504; 1980.

23. Turek, F. W. Circadian neural rhythms in mammals. Annu. Rev. Physiol. 47:49-64; 1985.

24. Turek, F. W.; Earnest, D. J.; Swann, J. Splitting of the circadian rhythm of activity in hamster. In: Aschoff, J.; Daan, S.; Groos, G. A., eds. Vertebrate circadian systems: Structure and physiology. New York: Springer; 1982:203-213.

25. Turek, F. W.: Gwinner, E. Role of hormones in the circadian organization of vertebrates. In: Achoff, J.; Daan, S.; Gross, G. A., eds. Vertebrate circadian systems: Structure and physiology. New York: Springer; 1982:173-182.

26. Wollnik, F. Sex differences in the daily pattern of locomotor activity in laboratory rats. Naturwissenschaften 72:158-160; 1985.

27. Wollnik, F,; Döhler, K.-D. Effects of adult or perinatal hormonal environment on ultradian rhythms in locomotor activity of laboratory LEW/Ztm rats. Physiol. Behav. 38:229-240; 1986

28. Wollnik, F.; Gärtner, K.; Büttner, D. Genetic analysis of circadian and ultradian locomotor activity rhythms in laboratory rats. Behav. Genet. 17:167-178; 1987.

29. Wollnik, F.; Turek, F. W. Effects of complete and partial SCN lesions on ultradian and circadian locomotor activity rhythms in LEW/Ztm rats. Soc. Neurosci. Abstr. 17:607; 1987.

30. Zucker, I. Hormones and hamster circadian organization. In: Suda, M.; Hayaishi, O.; Nakagawa, H., eds. Biological rhythms and their central mechanism. Elsevier: North-Holland Biomedical Press; 1979:369-381.

31. Zucker, I.; Fitzgerald, K. M.; Morin, L. P. Sex differentiation of the circadian system in the golden hamster. Am. J. Physiol. 238:R97-R101; 1980. 